\title{
Loneliness and Social Engagement in Older Adults: A Bivariate Dual Change Score Analysis
}

\author{
Joanna E. McHugh Power \\ Queen's University Belfast \\ Frank Kee \\ Queen's University Belfast
}

\author{
Andrew Steptoe \\ University College London \\ Brian A. Lawlor \\ Trinity College Dublin
}

\begin{abstract}
Few longitudinal studies have explored the impact of loneliness on social engagement. We investigated whether loneliness would result in decreased social engagement over time among older adults and also whether the converse, that low levels of social engagement would predict increases in loneliness, held. Additionally, we explored potential mechanisms (specifically, memory and depressive symptomatology as mediators) in the bidirectional relationship(s) between loneliness and social engagement. Data from 4,714 adults over 50 years of age, participating in Waves 3, 4, and 5 of the English Longitudinal Study of Ageing (between 2006 and 2011), were analyzed using bivariate dual change scores within structural equation models. Higher levels of loneliness were inversely associated with social engagement over time, and high levels of social engagement were inversely associated with loneliness over time. To address the 2nd aim, we used structural equation modeling to evaluate potential mediators of the bidirectional relationships between loneliness and changes in social engagement. Depressive symptomatology, semantic memory, and episodic memory were found to partially mediate the relationship between loneliness measured at baseline and social engagement 4 years later. In addition, these variables also partially mediated the relationship between social engagement at baseline and loneliness 4 years later. A comparison of the 2 models revealed that the model proposing a pathway from loneliness to social engagement (as mediated by depressive symptoms and memory) provided a better fit to the data. Implications for theories of loneliness are discussed.
\end{abstract}

Keywords: loneliness, aging, cognitive aging, memory

Supplemental materials: http://dx.doi.org/10.1037/pag0000287.supp

Loneliness, a felt insufficiency in the quality of social relationships, can present problems in later life (Pinquart \& Sorensen, 2001). Loneliness is problematic not just because it is an undesirable emotional state but also because of its associations with cognitive decline (Boss, Kang, \& Branson, 2015), dementia risk (Wilson et al., 2007), early mortality risk (Holt-Lunstad, Smith, Baker, Harris, \& Stephenson, 2015), and cardiovascular disease (Valtorta, Kanaan, Gilbody, Ronzi, \& Hanratty, 2016).

Given these associations, it is important to understand how best to intervene in individuals who are lonely. One prerequisite for the effective design of interventions is an understanding of the possible mechanisms (Wight, Wimbush, Jepson, \& Doi, 2015) through which the subject of intervention impacts health. In this context, it is important to identify an empirically validated theory of loneliness, its antecedents, and its consequences.

It is not surprising, then, that there have been many attempts to characterize loneliness (Perlman \& Peplau, 1982; Sønderby \& Wagoner, 2013) and to describe its antecedents and consequences. The influential theory of loneliness described by Weiss and others, for instance, describes loneliness as an innate force that promotes
This article was published Online First September 13, 2018.

Joanna E. McHugh Power, United Kingdom CRC Centre of Excellence for Public Health, Queen's University Belfast; Andrew Steptoe, Department of Epidemiology and Public Health, University College London; Frank Kee, United Kingdom CRC Centre of Excellence for Public Health, Queen's University Belfast; Brian A. Lawlor, Institute of Neuroscience, Trinity College Dublin.

Ideas appearing in this article were presented in a very preliminary form at the Alzheimer's Association International Conference, London, United Kingdom, in July 2016.
This work was conducted with support from the Centre for Ageing Research and Development in Ireland, in the form of a Leadership in Ageing Research Fellowship held by the corresponding author. We thank the funders for their support and also thank the research team at the English Longitudinal Study on Ageing (ELSA), as well as the participants of the study, and the United Kingdom Data Service at the United Kingdom Data Archive for facilitating access to the ELSA data set.

Correspondence concerning this article should be addressed to Joanna E. McHugh Power, who is now at the School of Business, National College of Ireland, Mayor Street, I.F.S.C., Dublin 1, Ireland. E-mail: mchughje@tcd.ie 
social engagement with others (Bowlby, 1973; Sullivan, 1953; Weiss, 1973). Similarly, Cacioppo and others have described loneliness as a biological drive activated because of social disengagement, designed to help the individual to return to a state of social homeostasis (e.g., Hawkley \& Cacioppo, 2010).

The manner in which loneliness is thought to promote social reengagement is further clarified in the social reconnection hypothesis (Maner, deWall, Baumeister, \& Schaller, 2007), the reaffiliation motive model (Qualter et al., 2015), and the model of belonging regulation (Gardner, Pickett, \& Brewer, 2000), all of which focus on the importance of behavioral and cognitive mechanisms. However, these models also describe the frequent failure of loneliness to engender social reengagement. In fact, social disengagement may be more likely to occur in the short term, with adaptive value, because it allows individuals to retreat, avoid further social rejection, and reflect on their social strategies (Cacioppo et al., 2015; Qualter et al., 2015).

According to these models, if attempts at social reengagement are made but are unsuccessful, the very cognitive and behavioral mechanisms normally driving reengagement may instead result in social disengagement (Qualter et al., 2015). It is possible that thwarted attempts at social reengagement elicit social anxiety, leading to an abandonment of these attempts and subsequent chronic loneliness (Cacioppo \& Hawkley, 2005; Cacioppo et al., 2006; Lucas, Knowles, Gardner, Molden, \& Jefferis, 2010). Alternatively, Gardner suggests that chronic loneliness develops because of behavioral deficits (Gardner, Pickett, Jefferis, \& Knowles, 2005). She describes three sequential stages of belonging regulation and posits that lonely individuals are generally successful at Stages 1 (assessing the current level of belonging need) and 2 (monitoring the social environment) but not 3 (initiating social engagement), which tends to lead to a lack of social reengagement and, subsequently, chronic loneliness (Gardner et al., 2005). As predicted, empirical demonstrations of the role of social disengagement in predicting loneliness are available (Dahlberg, Andersson, \& Lennartsson, 2018). However, less evidence is available demonstrating the impact of loneliness on social disengagement. Atop social anxiety and behavioral deficits, loneliness may lead to social disengagement via its other documented negative consequences on social functioning. Loneliness precedes dissatisfaction with social relationships (Hawkley, Preacher, \& Cacioppo, 2007), and individuals who are lonely display social avoidance (Nurmi, Toivonen, Salmela-Aro, \& Eronen, 1996) and negative social expectancies (Frankel \& Prentice-Dunn, 1990). Loneliness, as such, despite its theoretically adaptive impact when transient, might best be described as pathological (Fromm Reichmann, 1959, Sønderby \& Wagoner, 2013) when chronic.

The main aim of the current investigation was to explore whether loneliness predicts changes to social engagement and whether the converse was true. Taken together, the theories discussed thus far all appear to suggest that (a) loneliness could lead to social disengagement or reengagement, depending on its impact on cognitive and behavioral factors, and (b) low levels of social engagement, or social disengagement, are likely to lead to subsequent loneliness. Theoretically, the causal sequence of events would begin with initially low levels of social engagement or social disengagement, although this would be difficult to demonstrate empirically using data from a relatively short follow-up period in a cohort study, in which it would be difficult to pinpoint the beginning of a process of social disengagement.

A consideration of possible mechanisms through which loneliness and social engagement may influence each other would further characterize their relationship. We refer to a previous model of the social-cognitive impact of loneliness to consider potential mediators in this relationship. Cacioppo and Hawkley previously suggested that loneliness may constitute a risk factor for poorer overall cognitive performance, faster cognitive decline, poor executive functioning, depressive cognition-negative thoughts, sensitivity to social threat, and self-defeating social cognition biases (Cacioppo \& Hawkley, 2009).

A complete evaluation of this model would necessitate empirical testing of all listed mechanisms through which loneliness might impact social cognition (and subsequently promote social disengagement); however, it was not possible in the current study to explore all mechanisms. Thus, we have limited our focus to two mediators of interest: memory and depressive symptoms. Loneliness was previously shown to be related to memory (Shankar, Hamer, McMunn, \& Steptoe, 2013; Wilson et al., 2007), and memory decline may precede social disengagement (Ballard, 2010). The prodromal phase of cognitive impairment and dementia, which likely lasts for many years (Verghese et al., 2006), might drive an individual to disengage socially because social interaction becomes too challenging (Saczynski et al., 2006). Loneliness is also a known risk factor for depressive symptoms (Cacioppo, Hawkley, \& Thisted, 2010; Heikkinen \& Kauppinen, 2004), whereas depression is thought to precede social disengagement (Allen \& Badcock, 2003). Because we were interested in bidirectional associations between loneliness and social engagement, it appeared necessary to also evaluate whether depressive symptoms and memory mediated the relationship between social engagement at baseline and loneliness at follow-up, because social engagement is known to drive memory decline (Zunzunegui, Alvarado, Del Ser, \& Otero, 2003) and depressive symptoms (Glass, De Leon, Bassuk, \& Berkman, 2006) among older adults. Memory decline and depressive symptoms in turn may precede loneliness in this age group (Ayalon, Shiovitz-Ezra, \& Roziner, 2016; Dahlberg, Andersson, McKee, \& Lennartsson, 2015).

Hypothesis 1: Bidirectional paths exist between loneliness and social engagement.

Hypothesis 2: Depressive symptomatology and memory constitute mediators of the (bidirectional) relationship(s) between loneliness and social engagement.

\section{Method}

\section{Design}

The English Longitudinal Study on Ageing (ELSA) is a representative, prospective, longitudinal cohort study tracking the ageing of adults living in England from 2002 onward. ELSA commenced with a sample of 11,391 adults over 50 years of age, and participants are followed up every 2 years. All participants have given informed consent to participate in the study, and the study was approved by the local ethics committee (Taylor et al., 2007). Data collected in Waves 3 (during 2006 and 2007), 4 (during 2008 
and 2009), and 5 (during 2010 and 2011) are hereby analyzed (because not all of the variables of interest were collected in Waves 1 and 2).

\section{Participants}

Participants included in the current analyses were those over 50 years of age, recruited as core sample members (rather than family members of sample members), community-dwelling, and free of dementia at Wave 3 and who had given an interview directly to the research team (rather than through a proxy). ${ }^{1}$ Individuals from Waves 4 and 5 were included only if they had participated in Wave 3 and were community-dwelling and free of dementia and had given an interview directly to the research team. This led to a sample size of 4,714. Because of the inclusion of refreshment cohorts, it is difficult to estimate attrition rates in ELSA, but of the 8,811 participants engaged at Wave 3, some 7,595 were engaged by Wave 4 and 7,178 by Wave 5 (Banks, Nazroo, \& Steptoe, 2014). For the purposes of the subsequent analyses, Waves 3, 4, and 5, respectively, are hereafter referred to as Times 1, 2, and 3. ELSA data are available for download following service registration (www.ukdataservice.ac.uk). The study was approved through the National Research Ethics Service, and all participants gave informed consent.

\section{Measures}

Loneliness and covariates. Loneliness was measured in ELSA using three items from the UCLA Modified Loneliness Scale, which has previously demonstrated acceptable psychometric characteristics (Hughes, Waite, Hawkley, \& Cacioppo, 2004; Russell, 1996). These three items are "I feel left out," "I feel isolated," and "I lack companionship." In Wave 3, reliability was acceptable (Cronbach's alpha $=.84$ ), as it was in Waves 4 (Cronbach's alpha $=.83$ ) and 5 (Cronbach's alpha $=.83$ ). Covariates were age, sex, comorbidity count (comprising selfreported heart attack, chronic heart failure, lung disease, diabetes, cancer, leukemia-lymphoma, angina, heart murmur, arrhythmia, stroke), and count of functional limitations in activities of daily living (these included difficulty dressing, including putting on shoes and socks; difficulty walking across a room; difficulty bathing or showering; difficulty eating or cutting up food; difficulty getting in and out of bed; and difficulty using the toilet).

Mediators. The mediators of interest were depressive symptomatology, episodic memory, and semantic memory. Depressive symptomatology was measured using the eight-item version of the Center for Epidemiological Studies-Depression Scale (CESD-8; (Radloff, 1977). An item in this scale regarding loneliness was removed to avoid issues with discriminant validity. Verbal episodic memory was measured using performance from the word-list learning task (whereby participants are read a list of 10 words and asked to recite them both immediately and after a delay and where scores of both tasks are used to give an overall word-list learning score). Semantic memory was measured using a measure of verbal fluency, the animal-naming task, whereby participants must name as many animals as they can think of in $60 \mathrm{~s}$. This task is also considered a measure of executive function.

\section{Social Engagement}

Although social disengagement (or a reduction in social engagement) was the main outcome of interest, it is also possible that participants may increase their social engagement over time. Social engagement in a number of different social activities was recorded at Waves 3, 4 and 5. At each wave, participants reported whether they participated in the following:

1. Political parties, trade unions, or environmental groups;

2. Tenants' or residents' groups or neighborhood watch;

3. A church or other religious group;

4. A charitable organization;

5. An education, arts, or music group or evening classes;

6. A social club;

7. Sports clubs, gyms, or exercise classes; and

8. Any other organizations, clubs, or societies.

Scores of 1 indicating engagement and 0 indicating nonengagement for each item were added to yield a maximum score of 8 (Time 1: $M d n=2$ activities, Time 2: $M d n=1$; Time 3, $M d n=$ 1 ; range $=0-8$ for all waves).

The mean change in social engagement between Times 1 and 3 was $-.75(S D=1.27$, range $=-7-8)$, meaning that people on average declined in their social engagement across this time frame.

\section{Data Analysis}

For Hypothesis 1, bivariate dual latent change score modeling (McArdle et al., 2004) within a structural equation model (SEM) framework was used. This approach resolves many of the issues with the more common cross-lagged panel modeling approach (Hamaker, Kuiper, \& Grasman, 2015). Bivariate dual latent change scores allow the simultaneous longitudinal modeling of two latent variables over time, with the additional flexibility to explore feedback parameter (autoregressive) and coupling parameter (cross-lagged) pathways. Latent change scores represent some change in a variable between time points, by first measuring the variable at Time 2 with a factor loading fixed to 1 and then introducing a beta parameter, which allowed us to measure the impact of levels of the variable at Time 1 on levels at Time 2 (Kievit, Brandmaier, et al., 2017). They can also be described as "difference scores corrected for measurement error" (Hamaker et al., 2015, p. 107). Latent change can be parsed into constant and proportional effects (these are the dual effects; McArdle, 2009),

\footnotetext{
${ }^{1}$ A number of individuals were excluded: 1,388 because they were not part of the core sample; 232 because they gave an interview via a proxy; 51 because they were institutionalized and not community-dwelling; 58 because they had received a diagnosis of dementia; 2,590 because they were part of the Wave 4 "refreshment sample" (a new sample of additional participants added to the cohort during some waves); and 102 who were subsequently excluded because they did not fit the previously mentioned criteria during Waves 4 and 5 (i.e., new cases of dementia, new proxy interviews, new institutionalization).
} 
whereby the constant effect (slope) is a fixed parameter that represents global change across all time points, as a measure of overall change, and proportional change, which represents more local change in a variable (e.g., between Time 1 and Time 2) proportionate to the previous state of that variable (i.e., level at Time 1; Kievit, Brandmaier, et al., 2017). Both constant and proportional effects can be invariant, to allow systematic accumulation of changes in a variable over time (McArdle, 2009).

We implemented the model using the lavaan package in $\mathrm{R}$ (Rosseel, 2012) and based our script on published tutorials (Ghisletta \& McArdle, 2012; Kievit, Brandmaier, et al., 2017). In the current model, a linear global pattern of change was specified (by specifying a factor loading of 1 from the constant to the slope effect). In a bivariate dual-change-score model, a coupling parameter is specified to represent the time-dependent effects of one variable on the latent change in the other (McArdle, 2009). As a result, change in this model is a function of constant and proportional effects and of the preceding score on the coupled variable - in the current model, for instance, change in loneliness between Times 1 and 2 is a function of the constant effect (i.e., slope of loneliness across the three time points), proportional effect (i.e., loneliness at Time 1 specifically), and the coupled variable (i.e., social engagement at Time 1).

Because of violations of multivariate normality, the robust weighted least squares estimator was used in the models described. Loneliness and social engagement were specified as latent variables, and latent change scores were specified from scores at Wave 3 (baseline) with change modeled between Waves 3 and 4 and between Waves 4 and 5. Four alternative models were evaluated: first, a model with both coupling parameters fixed to 0; second, two subsequent models, each with only one directional coupling parameter fixed to 0 ; and a final model with bidirectional coupling parameters freely estimated (Quinn, 2012). Chi-square difference tests were used to compare these four models to evaluate the relative model improvement when coupling parameters were introduced. The covariates mentioned earlier were also included in all models (and in the subsequent mediation models).

For Hypothesis 2, mediation within a structural equation modeling framework was used. Linearity was checked in all relevant variable pairs prior to analyses and found to be satisfactory (see Table S1 in the online supplemental materials). As such, a maximum likelihood estimator was used in the mediation model, and full information maximum likelihood was used to impute missing data (Kline, 2005).

\section{Results}

\section{Sample Characteristics}

The sample are described at baseline (here being Wave 3, or Time 1) of the study in Table 1. Although the version of the CESD-8 Scale used for analyses was calculated without the item regarding loneliness, the version reported in Table 1 retains this item. In the measurement model component of the bivariate dual change score model, loneliness at each time point was regressed as a latent variable on three items measuring loneliness, with resulting factor loadings ranging between .47 and .58 across all three time points (see Figure 1). These constitute acceptable factor
Table 1

Baseline (i.e. Wave 3) Characteristics of 4,714 Individuals Over Age 50 in the Current Analysis

\begin{tabular}{|c|c|c|c|c|}
\hline Characteristic & $M(S D)$ & $M d n$ & $\%$ & Range \\
\hline Age & $65.8(8.4)$ & & & $50-99$ \\
\hline Sex (male) & & & 43 & \\
\hline Comorbidity count & .4 & 0 & & $0-4$ \\
\hline ADL functional limitations (count) & .29 & 0 & & $0-6$ \\
\hline CESD-8 $\operatorname{scores}^{\mathrm{a}}$ & $1.32(1.84)$ & & & $0-8$ \\
\hline UCLA Loneliness Scale & $2.15(2.23)$ & & & $0-10$ \\
\hline Wave 3 social activity engagement count & $2.13(1.38)$ & 2 & & $0-8$ \\
\hline Verbal fluency ${ }^{\mathrm{b}}$ & $20.97(6.46)$ & & & $0-56$ \\
\hline Episodic memory: Immediate recall & $6(1.67)$ & & & $0-10$ \\
\hline Episodic memory: Delayed recall & $4.82(1.96)$ & & & $0-10$ \\
\hline
\end{tabular}

Note. $\mathrm{ADL}=$ activities of daily living; CESD-8 = eight-item Center for Epidemiological Studies-Depression Scale; UCLA = University of California at Los Angeles; $M=$ Mean; $S D=$ Standard Deviation; $M d n=$ Median.

${ }^{a}$ Depressive symptomatology loneliness item retained. ${ }^{b}$ Based on an animal-naming task.

loadings and indicate that loneliness as a latent variable is acceptably measured using the three items.

Hypothesis 1. The first model to be implemented was the version with both bidirectional parameters fixed to 0. Model 1 had marginally acceptable fit and converged after 95 iterations; diagonally weighted least squares (DWLS) statistics: $\chi^{2}(106, N=$ $3773)=1,890.47, p<.001$, comparative fit index $(\mathrm{CFI})=.93$, Tucker-Lewis index $(\mathrm{TLI})=.92$, root-mean-square error of approximation $($ RMSEA) $=.07$, standardized root-mean-square residual $($ SRMR $)=.08$; robust statistics: $\chi^{2}(106, N=3773)=$ $2,014.02, p<.001, \mathrm{CFI}=.78, \mathrm{TLI}=.77, \mathrm{RMSEA}=.07$, SRMR $=.08$; scaling correction factor $=.96$, shift parameter $=$ 37.62 .

In Model 2, the path between loneliness and social engagement was fixed to 0 . The path between social engagement and loneliness was freely estimated. The model converged after 108 iterations. Model fit was again marginally acceptable, DWLS statistics: $\chi^{2}(105, N=3773)=1,878.33, p<.001, \mathrm{CFI}=.93, \mathrm{TLI}=.92$, RMSEA $=.07$, SRMR $=.08$; robust statistics: $\chi^{2}(106, N=$ $3773)=2,031.47, p<.001, \mathrm{CFI}=.78, \mathrm{TLI}=.76$, RMSEA $=$ $.07, \mathrm{SRMR}=.08$; scaling correction factor $=.942$, shift parameter $=36.64$. A chi-squared difference test comparing Models 1 and 2 indicated that Model 2 provided a better fit to the data, $\Delta \chi^{2}(1)=13.97, p<.001$.

In Model 3, the path between social engagement and loneliness was fixed to 0 . The path between loneliness and social engagement was freely estimated. The model converged after 106 iterations. Model fit was again marginally acceptable, DWLS statistics: $\chi^{2}(105, N=3773)=1,887.15, p<.001$, CFI $=.93$, TLI $=.92$, RMSEA $=.07$, SRMR $=.08$; robust statistics: $\chi^{2}(105, N=$ $3773)=2,006.62, p<.001, \mathrm{CFI}=.79, \mathrm{TLI}=.77, \mathrm{RMSEA}=$ .07, SRMR $=.08 ;$ scaling correction $=.96$, shift parameter $=$ 37.07. A chi-squared difference test comparing Models 1 and 3 indicated that there was a difference between the two in terms of model fit, $\Delta \chi^{2}(1)=5.84, p \leq .015$, such that Model 3 provided the better fit.

In Model 4, the final model, both bidirectional pathways were freely estimated. The model converged after 118 iterations. Model 


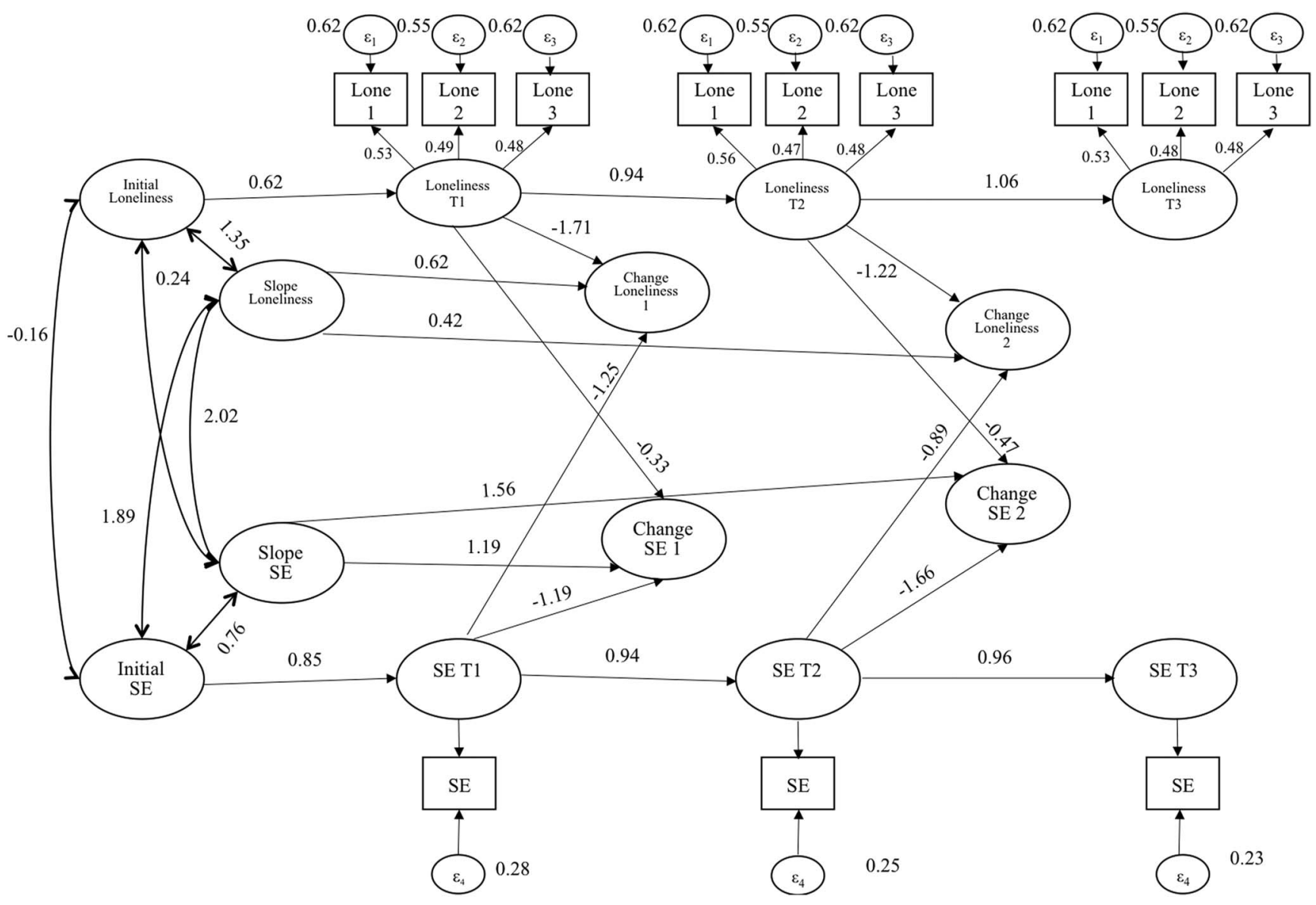

Figure 1. Bivariate dual change score model describing feedback and coupling parameters between loneliness and social engagement at three time points in the English Longitudinal Study on Ageing data (Waves 3, 4, and 5). Omitted from the image are covariates (age, sex, comorbidity count, activities of daily living limitation count), upon which the first latent change scores (of loneliness [Lone] and social engagement [SE]) were regressed. $\mathrm{T}=$ time.

fit was again marginally acceptable, DWLS statistics: $\chi^{2}(104, N=$ $3773)=1,857.59, p<.001, \mathrm{CFI}=.93, \mathrm{TLI}=.92, \mathrm{RMSEA}=$ .07, SRMR $=.08$; robust statistics: $\chi^{2}(104, N=3773)=$ $2,025.22, p<.001, \mathrm{CFI}=.78, \mathrm{TLI}=.76$, RMSEA $=.07$, SRMR $=.08 ;$ scaling correction $=.93$, shift parameter $=35.42$. The model parameters are given in Figure 1 (see also Table 2).

At Time 1, as expected, loneliness and social engagement had a negative correlation $(r=-.16, p<.001)$. Both coupling parameters were negative: Those individuals who at Time 1 had low levels of loneliness had higher improvements in social engagement over time, and vice versa. In the context of other parameters of change over time, including self-feedback and slope, the coupling parameter from social engagement to change in loneliness between Times 1 and 2 was negative $(\beta=-1.25, p<.001)$ and between social engagement at Time 2 and change in loneliness between Times 1 and 2 was also negative $(\beta=-.89, p<.001)$. The coupling parameter from loneliness at Time 1 to change in social engagement between Times 1 and 2 was negative $(\beta=-.33, p<.001)$ and between loneliness at Time 2 and changes in social engagement between Times 2 and 3 was also negative $(\beta=-.47, p<.001$; see Table 2 and Figure 1$)$.
Because coupling parameters must be interpreted in combination with other parameters of change (Lövdén, Ghisletta, \& Lindenberger, 2005), we plotted results on a vector field plot to elucidate the model results (see Figure 2), in the same manner as that used by Kievit and colleagues previously (Kievit, Lindenberger, et al., 2017). From this plot, limiting interpretation to the area inside the ellipse to capture only information within the $90 \%$ confidence interval $(\mathrm{CI})$ of the raw data, it is clear that low scores of loneliness at Time 1 exert a small positive effect on social engagement scores over time, relative to high scores of loneliness, which yield a small overall negative effect on social engagement scores over time. Low scores of social engagement at Time 1, meanwhile, have a negligible effect on loneliness over time, whereas high scores of social engagement at Time 1 exert a negative impact on loneliness over time.

A chi-squared difference test comparing Models 1 and 4 indicated that Model 4 provided a better fit to the data, $\Delta \chi^{2}(1)=$ $39.05, p<.001$. Model 4 was also an improvement over Models $2, \Delta \chi^{2}(1)=44.88, p<.001$, and $3, \Delta \chi^{2}(1)=31.14, p<.001$, suggesting that the model providing the best fit to the data is that 
Table 2

Bivariate Dual Change Score Model (i.e. Model 4)/ with Bidirectional Coupling Parameters Freely Estimated

\begin{tabular}{|c|c|c|c|c|}
\hline Regression path & $\beta$ & $S E$ & $z$ & $p$ \\
\hline \multicolumn{5}{|c|}{ Feedback parameters } \\
\hline Loneliness: $\mathrm{T} 1$ to $\mathrm{T} 2$ & .94 & & & \\
\hline Loneliness: $\mathrm{T} 2$ to $\mathrm{T} 3$ & 1.06 & & & \\
\hline Social engagement: $\mathrm{T} 1$ to $\mathrm{T} 2$ & .94 & & & \\
\hline Social engagement: $\mathrm{T} 2$ to $\mathrm{T} 3$ & .96 & & & \\
\hline \multicolumn{5}{|c|}{ Pathways to change in loneliness: $\mathrm{T} 1$ to $\mathrm{T} 2$} \\
\hline T1 loneliness & -1.71 & .04 & -9.84 & $<.001$ \\
\hline Age & .02 & .000 & .79 & .43 \\
\hline Sex & .53 & .02 & 7.68 & $<.001$ \\
\hline Comorbidities & .37 & .02 & 4.48 & $<.001$ \\
\hline ADL limitations & .55 & .02 & 6.36 & $<.001$ \\
\hline \multicolumn{5}{|c|}{ Pathways to change in loneliness: $\mathrm{T} 2$ to $\mathrm{T} 3$} \\
\hline T2 loneliness (feedback) & -1.22 & .04 & -9.84 & $<.001$ \\
\hline \multicolumn{5}{|c|}{ Pathways to change in social engagement: $\mathrm{T} 1$ to $\mathrm{T} 2$} \\
\hline T1 social engagement (feedback) & -1.19 & .08 & -7.43 & $<.001$ \\
\hline Age & -.04 & .001 & -2.63 & .009 \\
\hline Sex & -.03 & .05 & -.83 & .41 \\
\hline Comorbidities & -.08 & .04 & -2.14 & .033 \\
\hline ADL limitations & -.21 & .03 & -5.40 & $<.001$ \\
\hline \multicolumn{5}{|c|}{ Pathways to change in social engagement: $\mathrm{T} 2$ to $\mathrm{T} 3$} \\
\hline T2 social engagement (feedback) & -1.66 & .08 & -7.43 & $<.001$ \\
\hline \multicolumn{5}{|l|}{ Coupling parameters } \\
\hline $\mathrm{T} 1$ social engagement to change in loneliness $\mathrm{T} 1$ to $\mathrm{T} 2$ & -1.25 & .02 & -6.67 & $<.001$ \\
\hline $\mathrm{T} 2$ social engagement to change in loneliness $\mathrm{T} 2$ to $\mathrm{T} 3$ & -.89 & .02 & -6.67 & $<.001$ \\
\hline $\mathrm{T} 1$ loneliness to change in social engagement $\mathrm{T} 1$ to $\mathrm{T} 2$ & -.33 & .06 & -7.32 & $<.001$ \\
\hline $\mathrm{T} 2$ loneliness to change in social engagement $\mathrm{T} 2$ to $\mathrm{T} 3$ & -.47 & .06 & -7.32 & $<.001$ \\
\hline
\end{tabular}

Note. $\mathrm{SEM}=$ structural equation model; $\mathrm{T}$ (in $\mathrm{T} 1-\mathrm{T} 3)=$ time; $\mathrm{ADL}=$ activities of daily living; $S E=$ Standard Error.

which includes the existence of bidirectional effects between loneliness and social engagement.

Hypothesis 2. Mediation analyses were conducted to see whether the relationship between loneliness at Time 1 and social engagement at Time 3 was mediated by episodic memory, semantic memory, and depressive symptomatology (measured at Time 2 ). The model converged after 92 iterations, and fit was borderline acceptable, $\chi^{2}(105, N=4216)=2,453, p<.001$, CFI $=.87$, $\mathrm{TLI}=.84, \mathrm{RMSEA}=.07, \mathrm{SRMR}=.07$; Akaike information criterion $(\mathrm{AIC})=152,080$; sample size-adjusted Bayesian information criterion $(\mathrm{ssBIC})=152,200$ (see Table S2 in the online supplemental materials for the measurement model).

In the structural model, higher levels of loneliness at Time 1 predicted lower levels of social engagement at Time $3(\beta=-.07$, $95 \%$ CI $[-.15,-.04]$; all reported confidence intervals are biascorrected asymmetric confidence intervals). Higher scores on episodic memory $(\beta=.17,95 \%$ CI $[.18, .29])$, lower scores on depressive symptomatology $(\beta=-.05,95 \%$ CI $[-.11,-.01])$, and higher scores on semantic memory $(\beta=.09,95 \%$ CI $[.01$, $.026]$ ), all at Time 2 , also predicted higher levels of social engagement at Time 3.

Higher levels of loneliness at Time 1 also predicted lower scores on episodic memory $(\beta=-.09,95 \% \mathrm{CI}[-.13,-.06], p<.001)$, higher scores on depressive symptomatology $(\beta=.47,95 \% \mathrm{CI}$
$[.47, .56])$, and lower scores on semantic memory $(\beta=-.09,95 \%$ CI $[-.80,-.38]$; see Table 3$)$, all at Time 2.

Results indicated the presence of mediation effects through episodic memory $(\beta=-.016, p<.001)$, depressive symptomatology $(\beta=-.02, p=.016)$, and semantic memory $(\beta=-.008$, $p<.01$; see Figure 3 ).

To rule out feasible alternatives, we explored a competing model to investigate whether social engagement mediated the relationship between loneliness and (a) episodic memory, (b) semantic memory, and (c) depression. Model fit was borderline acceptable, $\chi^{2}(105, N=4205)=2,555, p<.001, \mathrm{CFI}=.87$, $\mathrm{TLI}=.83, \mathrm{RMSEA}=.07, \mathrm{SRMR}=.07 ; \mathrm{AIC}=153,094$, ssBIC $=153,214$. This indicates that the initial model, positing that episodic memory, semantic memory, and depression mediate the relationship between loneliness and social engagement, fit the data better.

An alternative model, exploring the potential for episodic memory, depressive symptomatology, and semantic memory to mediate the relationship between social engagement at Time 1 and loneliness at Time 3, was also derived and demonstrated marginally acceptable fit, converging after 90 iterations, $\chi^{2}(105, N=4314)=$ $2,713.38, p<.001, \mathrm{CFI}=.86, \mathrm{TLI}=.83$, RMSEA $=.08$, SRMR $=.07 ;$ AIC $=155,092$, ssBIC $=155,214$. 


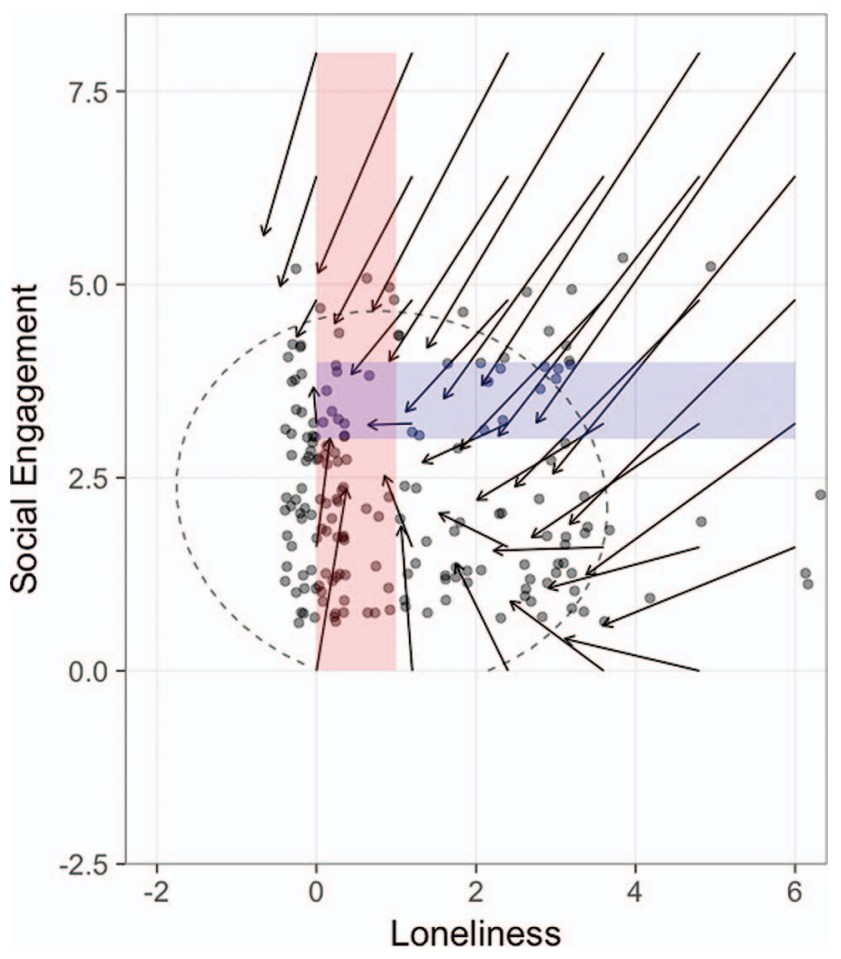

Figure 2. Vector field plot for the bivariate dual change score model with both coupling parameters freely estimated (i.e., Model 4). Model-implied changes are indicated by arrows, and raw data represented by dots. The dots represent the scores of loneliness and social engagement at Time 1 for a random subset of individuals. Each arrow represents model-implied change between Time 1 (the base of the arrow) and Time 2 (the head of the arrow). The horizontal shaded area elucidates the impact of social engagement scores on change in loneliness over time, whereas the vertical area elucidates the impact of loneliness on change in social engagement scores over time. The dashed ellipse shows the $90 \%$ confidence interval for the raw data. See the online article for the color version of this figure.

In the structural model, higher levels of social engagement at Time 1 predicted lower levels of loneliness at Time $3(\beta=-.05$, $p=.001)$. Lower levels of episodic memory $(\beta=-.06, p=$ $.004)$, higher levels of depressive symptomatology $(\beta=.42, p<$ .001 ), and (nonsignificant) lower levels of semantic memory $(\beta=-.03, p=.076)$, all at Time 2 , also predicted higher levels of loneliness at Time 3 .

Higher levels of social engagement at Time 1 also predicted higher scores on episodic memory $(\beta=.18, p<.001)$, lower scores on depressive symptomatology $(\beta=-.11, p<.001)$, and higher scores on semantic memory $(\beta=.16, p<.001)$, all at Time 2 . Results indicated the presence of mediation effects through episodic memory $(\beta=-.016, p=.001)$, depressive symptomatology $(\beta=-.02, p=.016)$, and semantic memory $(\beta=-.008$, $p=.006)$.

Based on the AIC and BIC figures, the model specifying that loneliness at Time 1 leads to social engagement at Time 3, as mediated by depressive symptomatology and episodic and semantic memory, fit better than did the oppositely specified model (with social engagement at Time 1 predicting loneliness at Time 3). We also included a competing model to this second model, hypothe- sizing that loneliness would mediate the relationships between social engagement and (a) episodic memory, (b) semantic memory, and (c) depression. Model fit was again borderline acceptable, $\chi^{2}(105, N=4816)=2,720, p<.001, \mathrm{CFI}=.86, \mathrm{TLI}=.82$, $\mathrm{RMSEA}=.08, \mathrm{SRMR}=.08 ; \mathrm{AIC}=152,330, \mathrm{ssBIC}=152,450$. This means that the competing model was preferential to the original Model 2 but did not have as good a fit as did the initial Model 1, which tested the hypothesis that episodic memory, semantic memory, and depression mediate the relationship between loneliness and social engagement: The model testing this hypothesis is the one that fit the data best.

\section{Discussion}

The current study aimed to test two hypotheses, using data from the English Longitudinal Study of Ageing. First, we hypothesized that there would be bidirectional effects between loneliness and social engagement. We found that higher levels of loneliness predicted a decrease in social engagement over time and that higher levels of social engagement predicted decreases in loneliness. Specifically, the effect of loneliness on social engagement was strongest at low levels of loneliness, where the effect was positive, and less strong at high levels of loneliness, where the effect was negative. Additionally, the effect of social engagement on change in loneliness over time was negligible at low levels of social engagement, but higher scores of social engagement at baseline had a negative impact on change in loneliness over time. The model that included both bidirectional effects fit the data best, and there was some evidence that the pathway from social engagement to changes in loneliness was marginally dominant over the reciprocal pathway.

We also hypothesized that episodic memory, semantic memory, and depressive symptomatology would mediate the relationship between loneliness and social engagement over time. This hypothesis was supported. Episodic and semantic memory partially suppressed the negative impact of loneliness on social engagement (because they both predicted increases in social engagement over time). Depressive symptomatology partially accounted for the overall negative relationship between loneliness and social engagement (because both loneliness and depressive symptomatology predicted decreases in social engagement over time). We tested three alternative hypotheses (which is strongly advocated when using SEM). Although both models were of borderline acceptable fit, Model 1, describing pathways from loneliness through mediators to social engagement, fit the data best. As such, depressive symptoms and memory are more likely to represent mechanisms through which loneliness impacts social engagement, rather than vice versa and rather than loneliness or social engagement representing mediators across the same variables. Further work is required to elucidate potential mechanisms through which social engagement impacts loneliness.

The study is not without limitations. These mediators represent only a subset of the likely pathways through which loneliness and social engagement impact one another, and alternatives have been suggested before (Cacioppo \& Hawkley, 2009). Additionally, one of the mediators (verbal fluency) is described as a measure of both executive function and semantic memory, so it is possible that our results could be interpreted to mean that executive functioning mediates the bidirectional associations between loneliness and 
Table 3

Structural Component of Mediation Model: Depression and Memory Mediate the Relationship between Loneliness and Social Engagement Predicting Social Engagement at (T3), Mediated by Depression, Episodic Memory, and Semantic Memory, (T2)

\begin{tabular}{|c|c|c|c|c|c|c|}
\hline Regression path & $\beta$ & $S E$ & $z$ & $p$ & $b$ & $95 \% C I^{\mathrm{a}}$ \\
\hline \multicolumn{7}{|c|}{ Pathways to T3 social engagement } \\
\hline T1 loneliness & -.07 & .03 & -3.62 & $<.001$ & -.09 & {$[-.15,-.04]$} \\
\hline T2 episodic memory & .17 & .03 & 8.69 & $<.001$ & .24 & {$[.18, .29]$} \\
\hline $\mathrm{T} 2$ semantic memory & .09 & .004 & 4.99 & $<.001$ & .02 & {$[.01, .026]$} \\
\hline $\mathrm{T} 2$ depression & -.05 & .03 & -2.41 & .016 & -.06 & {$[-.11,-.011]$} \\
\hline \multicolumn{7}{|c|}{ Pathways from $\mathrm{T} 1$ loneliness } \\
\hline T2 episodic memory & -.09 & .02 & -5.13 & $<.001$ & -.09 & {$[-.13,-.06]$} \\
\hline $\mathrm{T} 2$ semantic memory & -.09 & .11 & -5.39 & .003 & -.59 & {$[-.80,-.38]$} \\
\hline $\mathrm{T} 2$ depression & .47 & .02 & 23.54 & $<.001$ & .52 & {$[.47, .56]$} \\
\hline \multicolumn{7}{|c|}{ Pathways to T1 loneliness (exogenous covariates) } \\
\hline Age & .03 & .002 & 1.69 & .092 & .004 & {$[-.001, .008]$} \\
\hline Sex & .14 & .03 & 8.35 & $<.001$ & .29 & {$[.22, .36]$} \\
\hline Comorbidities & .08 & .03 & 4.41 & $<.001$ & .12 & {$[.07, .17]$} \\
\hline \multicolumn{7}{|l|}{ ADL limitations } \\
\hline Indirect effects & .16 & .02 & .53 & $<.001$ & .22 & {$[.17, .26]$} \\
\hline Total effect & -.117 & .02 & -6.95 & .001 & -.16 & {$[-.21,-.12]$} \\
\hline Sum of indirect effects & -.05 & .01 & -4.56 & .001 & -.06 & {$[-.09,-.04]$} \\
\hline Episodic memory: Indirect effect & -.02 & .01 & -4.46 & .001 & -.02 & {$[-.01,-.022]$} \\
\hline Depressive symptomatology: Indirect effect & -.02 & .01 & -2.41 & .016 & -.03 & {$[-.056,-.006]$} \\
\hline Semantic memory: Indirect effect & -.01 & .003 & -3.68 & .001 & -.011 & {$[-.017,-.005]$} \\
\hline
\end{tabular}

Note. $\quad S E M=$ structural equation model; $\mathrm{T}($ in $\mathrm{T} 1-\mathrm{T} 3)=$ time; $C I=$ confidence interval; $\mathrm{ADL}=$ activities of daily living; $S E=$ Standard Error; $C I=$ Confidence Intervals.

${ }^{a}$ Derived using bias-corrected bootstrapping.

social engagement. Because we did not have the data to examine all alternatives, we recommend that future research do so.

Effect sizes were small, with two exceptions. First, in the model without coupling parameters added, there was a moderately sized autoregressive effect of loneliness on later measures of loneliness. This implies that loneliness remains quite stable over time and that the best predictor of loneliness is past loneliness. Second, there was a moderately sized effect of loneliness at Time 1 on depressive symptomatology at Time 2, corroborating previous descriptions of loneliness as a serious risk factor for depression in later life (Cacioppo et al., 2010).

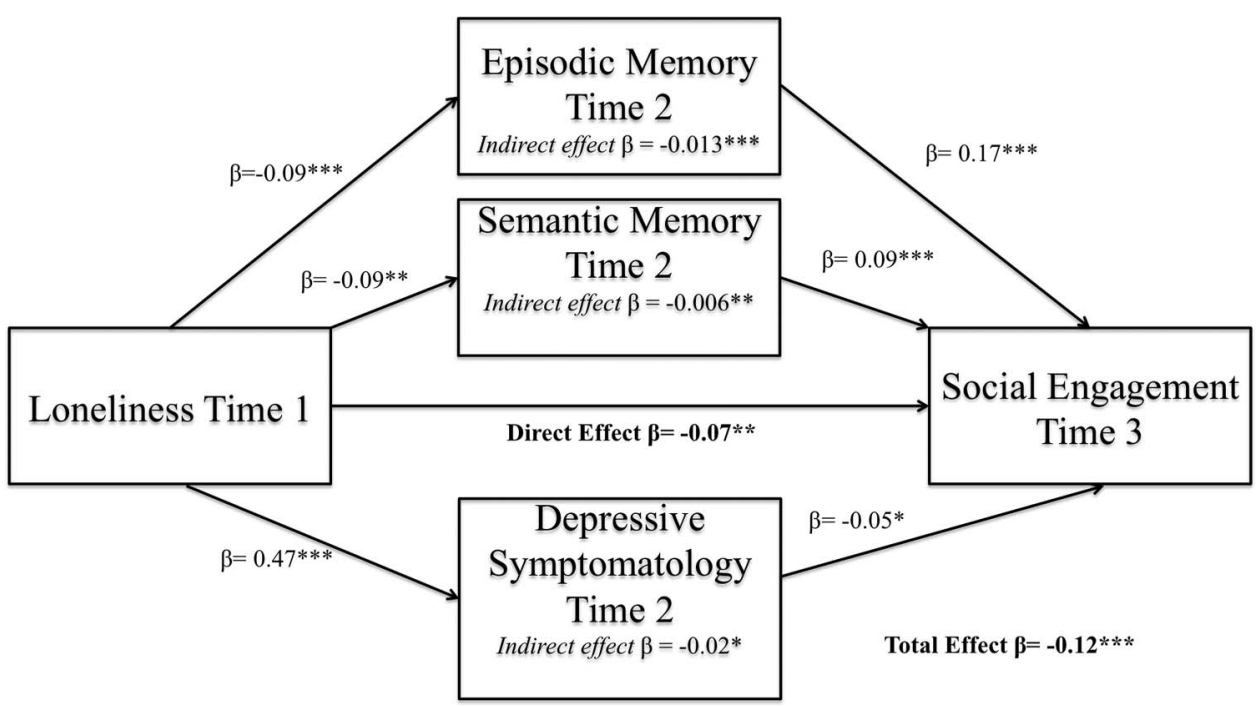

Figure 3. Mediation model investigated for Hypothesis 3: The mediating effects of episodic and semantic memory, and of depressive symptomatology, in the relationship between loneliness and social engagement over time. ${ }^{*} p<.05 .{ }^{* * *} p<.01 .{ }^{* * *} p<.001$. 
Loneliness had a small association with social engagement over time, such that higher levels of loneliness were related to a decrease in social engagement. In SEM analysis we observed that higher levels of loneliness at Time 1 were also related to lower levels of social engagement at Time 3. As such, high levels of loneliness preceded social disengagement over a period of 4 years, which, depending on the definition of short-term, corroborates Cacioppo's prediction that in the short term, loneliness leads to (possibly adaptive) social disengagement (Cacioppo et al., 2015). Alternatively, it is possible that these findings challenge the theory that high levels of loneliness precede social reengagement (Weiss, 1973), although this may take a longer time frame to manifest. Additionally, upon further inspection, the majority of the effect of loneliness on social engagement was accounted for by the mediating presence of depressive symptomatology. Thus, the independent effect that loneliness exerts on social engagement is minimal.

Incidentally, although short- and long-term effects of loneliness are discussed in the literature, there appears to be little consensus on their definitions. Loneliness is sometimes measured in terms of daily fluctuations, which would suggest that short-term loneliness operates on the order of days, whereas long-term loneliness could constitute loneliness lasting years. Further consideration of these definitions in future research is warranted.

Social engagement also predicts changes in loneliness over time, such that higher levels of social engagement predict a decrease in loneliness. Additionally, in the SEM analysis, high levels of social engagement at Time 1 were related to lower levels of loneliness at Time 3, findings that accord with the social needs perspective on loneliness - that it is experienced in the absence of sufficient social contact, which serves various relational provisions (Weiss, 1974).

Previous descriptions of loneliness have indicated that when transient, it promotes social engagement (Bowlby, 1973; Cacioppo et al., 2006; Sullivan, 1953; Weiss, 1973). This was not found to be the case in the current analysis. Broadly, our results are consistent with a description of loneliness as a pathological, rather than a functional, state. As loneliness increased, changes in social engagement decreased or became negative in nature (i.e., social engagement decreased). Thus, our results provide evidence that loneliness is a risk factor for social disengagement.

However, it is possible that this discordance between earlier theory and current results is due to the way loneliness is measured in our current study - we did not measure transient loneliness. In fact, the scale that was used in the current study is thought to measure chronic loneliness specifically (Queen, Stawski, Ryan, \& Smith, 2014). Future research comparing the effects of transient and chronic loneliness on social disengagement or reengagement would be crucial to further elucidate the nature of loneliness and whether it can be characterized as functional or pathological, or both, in different time frames.

Social engagement in the current study was measured as a count of engagement, at least monthly, in a series of relatively formal activities. Many of these activities are only incidentally social. This may not be the best way to measure social activity engagement. Previous studies of social disengagement have operationalized the variable in different ways: for instance, as the number of social ties an individual has (to a spouse, relatives, and friends; Bassuk, Glass, \& Berkman, 1999). However, it is likely that formal modes of social activity engagement may be the first to be discontinued if an individual is in a process of social disengagement and that engagement with more proximate social links (e.g., family members and close friends) would persist for longer.

Our method of data analysis represents a robust and flexible approach to evaluating relationships between observed and latent variables over time. The bivariate latent change score approach to analyzing suspected cross-lagged relations resolves many of the issues previously highlighted with the more traditional crosslagged panel modeling approach (Hamaker et al., 2015), and its ability to evaluate sophisticated hypotheses about change mean that it is a valuable approach for those interested in studying developmental trajectories (Kievit, Brandmaier, et al., 2017) and, by the same token, expected changes in later life.

Although SEM represents a robust, flexible analytic approach, evaluating mediation in this manner is still limited regarding causal inference. Counterfactual approaches to causal inference are gaining traction in investigations of mediation in the social sciences (De Stavola, Daniel, Ploubidis, \& Micali, 2015).

Episodic and semantic memory, and depressive symptomatology, were found to be significant mediators of the relationship between loneliness and social engagement. This suggests that loneliness in part exerts its effect on social disengagement via memory and depression. Our results accord with the significant existing literature linking loneliness to aspects of cognitive functioning (Boss et al., 2015), including semantic memory (Wilson et al., 2007), verbal episodic memory (Shankar et al., 2013), and depression (Cacioppo et al., 2010; Heikkinen \& Kauppinen, 2004). Loneliness itself is an undesirable and painful experience that warrants intervention in its own right, but aside from this, current results are consistent with the possibility that reducing loneliness might in turn reduce levels of social disengagement and depressive symptomatology and would potentially protect memory functioning in later life. Because the current analyses are based upon observational data only, further experimental research is required to test what for now are causal conjectures.

In summary, we report that loneliness is associated with disengagement in social activities over time, that the converse was also found to be the case, and that the bidirectional relationships between loneliness and social engagement are mediated in part by depressive symptomatology, as well as by verbal and semantic memory. Results have implications for theories of loneliness, because loneliness was observed to predict social disengagement, rather than reengagement, which fits with some of the predictions by Cacioppo (Cacioppo et al., 2015) but not by Weiss (Weiss, 1973), although temporality may be a factor in this prediction, with chronic loneliness predicting disengagement because transient loneliness predicts reengagement. Alternatively, loneliness may be characterized as a mostly pathological phenomenon, contrary to descriptions of its adaptive value (Cacioppo et al., 2015; Qualter et al., 2015). Results have clinical implications for those aiming to reduce cognitive decline, depression, and social disengagement among older adults.

\section{References}

Allen, N. B., \& Badcock, P. B. (2003). The social risk hypothesis of depressed mood: Evolutionary, psychosocial, and neurobiological perspectives. Psychological Bulletin, 129, 887-913. http://dx.doi.org/10 .1037/0033-2909.129.6.887

Ayalon, L., Shiovitz-Ezra, S., \& Roziner, I. (2016). A cross-lagged model of the reciprocal associations of loneliness and memory functioning. 
Psychology and Aging, 31, 255-261. http://dx.doi.org/10.1037/pag 0000075

Ballard, J. (2010). Forgetfulness and older adults: Concept analysis. Journal of Advanced Nursing, 66, 1409-1419. http://dx.doi.org/10.1111/j 1365-2648.2010.05279.x

Banks, J., Nazroo, J., \& Steptoe, A. (Eds.). (2014). The dynamics of ageing: Evidence from the English Longitudinal Study of Ageing 2002-12 (Wave 6). Retrieved from https://www.ifs.org.uk/publications/ 8696

Bassuk, S. S., Glass, T. A., \& Berkman, L. F. (1999). Social disengagement and incident cognitive decline in community-dwelling elderly persons. Annals of Internal Medicine, 131, 165-173.

Boss, L., Kang, D. H., \& Branson, S. (2015). Loneliness and cognitive function in the older adult: A systematic review. International Psychogeriatrics, 27, 541-553. http://dx.doi.org/10.1017/S1041610214002749

Bowlby, J. (1973). Attachment and Loss (Vol. 2). New York, NY: Basic Books.

Cacioppo, J. T., Cacioppo, S., Cole, S. W., Capitanio, J. P., Goossens, L., \& Boomsma, D. I. (2015). Loneliness across phylogeny and a call for comparative studies and animal models. Perspectives on Psychological Science, 10, 202-212. http://dx.doi.org/10.1177/1745691614564876

Cacioppo, J. T., \& Hawkley, L. C. (2005). People thinking about people: The vicious cycle of being a social outcast in one's own mind. In K. P. Williams, J. P. Forgas, \& W. von Hippel (Eds.), The social outcast: Ostracism, social exclusion, rejection, and bullying (pp. 91-108). New York, NY: Psychology Press.

Cacioppo, J. T., \& Hawkley, L. C. (2009). Perceived social isolation and cognition. Trends in Cognitive Sciences, 13, 447-454. http://dx.doi.org/ 10.1016/j.tics.2009.06.005

Cacioppo, J. T., Hawkley, L. C., Ernst, J. M., Burleson, M. H., Berntson, G. G., Nouriani, B., \& Spiegel, D. (2006). Loneliness within a nomological net: An evolutionary perspective. Journal of Research in Personality, 40, 1054-1085. http://dx.doi.org/10.1016/j.jrp.2005.11.007

Cacioppo, J. T., Hawkley, L. C., \& Thisted, R. A. (2010). Perceived social isolation makes me sad: 5-year cross-lagged analyses of loneliness and depressive symptomatology in the Chicago Health, Aging, and Social Relations Study. Psychology and Aging, 25, 453-463. http://dx.doi.org/ 10.1037/a0017216

Dahlberg, L., Andersson, L., \& Lennartsson, C. (2018). Long-term predictors of loneliness in old age: Results of a 20-year national study. Aging \& Mental Health, 22, 190-196. http://dx.doi.org/10.1080/13607863 .2016 .1247425

Dahlberg, L., Andersson, L., McKee, K. J., \& Lennartsson, C. (2015). Predictors of loneliness among older women and men in Sweden: A national longitudinal study. Aging \& Mental Health, 19, 409-417. http://dx.doi.org/10.1080/13607863.2014.944091

De Stavola, B. L., Daniel, R. M., Ploubidis, G. B., \& Micali, N. (2015). Mediation analysis with intermediate confounding: Structural equation modeling viewed through the causal inference lens. American Journal of Epidemiology, 181, 64-80. http://dx.doi.org/10.1093/aje/kwu239

Frankel, A., \& Prentice-Dunn, S. (1990). Loneliness and the processing of self-relevant information. Journal of Social and Clinical Psychology, 9, 303-315. http://dx.doi.org/10.1521/jscp.1990.9.3.303

Fromm Reichmann, F. (1959). Loneliness. Psychiatry: Interpersonal and Biological Processes, 22, 1-15. http://dx.doi.org/10.1080/00332747 .1959 .11023153

Gardner, W. L., Pickett, C. L., \& Brewer, M. B. (2000). Social exclusion and selective memory: How the need to belong influences memory for social events. Personality and Social Psychology Bulletin, 26, 486-496. http://dx.doi.org/10.1177/0146167200266007

Gardner, W. L., Pickett, C. L., Jefferis, V., \& Knowles, M. (2005). On the outside looking in: Loneliness and social monitoring. Personality and Social Psychology Bulletin, 31, 1549-1560. http://dx.doi.org/10.1177/ 0146167205277208
Ghisletta, P., \& McArdle, J. J. (2012). Latent curve models and latent change score models estimated in R. Structural Equation Modeling, 19, 651-682. http://dx.doi.org/10.1080/10705511.2012.713275

Glass, T. A., De Leon, C. F. M., Bassuk, S. S., \& Berkman, L. F. (2006). Social engagement and depressive symptoms in late life: Longitudinal findings. Journal of Aging and Health, 18, 604-628. http://dx.doi.org/ 10.1177/0898264306291017

Hamaker, E. L., Kuiper, R. M., \& Grasman, R. P. (2015). A critique of the cross-lagged panel model. Psychological Methods, 20, 102-116. http:// dx.doi.org/10.1037/a0038889

Hawkley, L. C., \& Cacioppo, J. T. (2010). Loneliness matters: A theoretical and empirical review of consequences and mechanisms. Annals of Behavioral Medicine, 40, 218-227. http://dx.doi.org/10.1007/s12160010-9210-8

Hawkley, L. C., Preacher, K. J., \& Cacioppo, J. T. (2007). Multilevel modeling of social interactions and mood in lonely and socially connected individuals: The MacArthur Social Neuroscience Studies. In A. D. Ong \& M. van Dulmen (Eds.), Oxford handbook of methods in positive psychology (pp. 559-575). New York, NY: Oxford University Press.

Heikkinen, R. L., \& Kauppinen, M. (2004). Depressive symptoms in late life: A 10-year follow-up. Archives of Gerontology and Geriatrics, 38, 239-250. http://dx.doi.org/10.1016/j.archger.2003.10.004

Holt-Lunstad, J., Smith, T. B., Baker, M., Harris, T., \& Stephenson, D. (2015). Loneliness and social isolation as risk factors for mortality: A meta-analytic review. Perspectives on Psychological Science, 10, 227 237. http://dx.doi.org/10.1177/1745691614568352

Hughes, M. E., Waite, L. J., Hawkley, L. C., \& Cacioppo, J. T. (2004). A short scale for measuring loneliness in large surveys: Results from two population-based studies. Research on Aging, 26, 655-672. http://dx.doi .org/10.1177/0164027504268574

Kievit, R. A., Brandmaier, A. M., Ziegler, G., van Harmelen, A. L., de Mooij, S. M. M., Moutoussis, M., . . . Dolan, R. J. (2017). Developmental cognitive neuroscience using latent change score models: A tutorial and applications. Developmental Cognitive Neuroscience. Advance online publication. http://dx.doi.org/10.1016/j.dcn.2017.11.007

Kievit, R. A., Lindenberger, U., Goodyer, I. M., Jones, P. B., Fonagy, P., Bullmore, E. T., . . . Dolan, R. J. (2017). Mutualistic coupling between vocabulary and reasoning supports cognitive development during late adolescence and early adulthood. Psychological Science, 28, 14191431. http://dx.doi.org/10.1177/0956797617710785

Kline, R. (2005). Principles and practice of structural equation modelling. New York, NY: Guilford Press.

Lövdén, M., Ghisletta, P., \& Lindenberger, U. (2005). Social participation attenuates decline in perceptual speed in old and very old age. Psychology and Aging, 20, 423-434. http://dx.doi.org/10.1037/0882-7974.20.3 423

Lucas, G. M., Knowles, M. L., Gardner, W. L., Molden, D. C., \& Jefferis, V. E. (2010). Increasing social engagement among lonely individuals: The role of acceptance cues and promotion motivations. Personality and Social Psychology Bulletin, 36, 1346-1359. http://dx.doi.org/10.1177/ 0146167210382662

Maner, J. K., DeWall, C. N., Baumeister, R. F., \& Schaller, M. (2007). Does social exclusion motivate interpersonal reconnection? Resolving the "porcupine problem." Journal of Personality and Social Psychology, 92, 42-55. http://dx.doi.org/10.1037/0022-3514.92.1.42

McArdle, J. J. (2009). Latent variable modeling of differences and changes with longitudinal data. Annual Review of Psychology, 60, 577-605. http://dx.doi.org/10.1146/annurev.psych.60.110707.163612

McArdle, J. J., Hamgami, F., Jones, K., Jolesz, F., Kikinis, R., Spiro, A., III, \& Albert, M. S. (2004). Structural modeling of dynamic changes in memory and brain structure using longitudinal data from the Normative Aging Study. Journals of Gerontology: Series B, Psychological Sciences 
and Social Sciences, 59, P294-P304. http://dx.doi.org/10.1093/geronb/ 59.6.P294

Nurmi, J. E., Toivonen, S., Salmela-Aro, K., \& Eronen, S. (1996). Optimistic, approach-oriented, and avoidance strategies in social situations: Three studies on loneliness and peer relationships. European Journal of Personality, 10, 201-219. http://dx.doi.org/10.1002/(SICI)1099-0984 (199609) 10:3<201::AID-PER257>3.0.CO;2-\#

Perlman, D., \& Peplau, L. A. (1982). Theoretical approaches to loneliness. In L. A. Peplau \& D. Perlman (Eds.), Loneliness: A sourcebook of current theory, research and therapy (pp. 123-134). New York, NY: Wiley-Interscience.

Pinquart, M., \& Sorensen, S. (2001). Influences on loneliness in older adults: A meta-analysis. Basic and Applied Social Psychology, 23, 245-266. http://dx.doi.org/10.1207/S15324834BASP2304_2

Qualter, P., Vanhalst, J., Harris, R., Van Roekel, E., Lodder, G., Bangee, M., . . Verhagen, M. (2015). Loneliness across the life span. Perspectives on Psychological Science, 10, 250-264. http://dx.doi.org/10.1177/ 1745691615568999

Queen, T. L., Stawski, R. S., Ryan, L. H., \& Smith, J. (2014). Loneliness in a day: Activity engagement, time alone, and experienced emotions. Psychology and Aging, 29, 297-305. http://dx.doi.org/10.1037/a003 6889

Quinn, J. (2012). Latent change score modeling of developmental relations between vocabulary and reading comprehension (Unpublished master's thesis). Department of Psychology, Florida State University, Tallahassee, Florida.

Radloff, L. (1977). The CES-D Scale: A self report depression scale for research in the general population. Applied Psychological Measurement, 1, 385-401. http://dx.doi.org/10.1177/014662167700100306

Rosseel, Y. (2012). lavaan: An R package for structural equation modeling. Journal of Statistical Software, 48, 1-36. http://dx.doi.org/10.18637/jss .v048.i02

Russell, D. W. (1996). UCLA Loneliness Scale (Version 3): Reliability, validity, and factor structure. Journal of Personality Assessment, 66, 20-40. http://dx.doi.org/10.1207/s15327752jpa6601_2

Saczynski, J. S., Pfeifer, L. A., Masaki, K., Korf, E. S., Laurin, D., White, L., \& Launer, L. J. (2006). The effect of social engagement on incident dementia: The Honolulu-Asia Aging Study. American Journal of Epidemiology, 163, 433-440. http://dx.doi.org/10.1093/aje/kwj061

Shankar, A., Hamer, M., McMunn, A., \& Steptoe, A. (2013). Social isolation and loneliness: Relationships with cognitive function during 4 years of follow-up in the English Longitudinal Study of Ageing. Psychosomatic Medicine, 75, 161-170. http://dx.doi.org/10.1097/PSY $.0 \mathrm{~b} 013 \mathrm{e} 31827 \mathrm{f09cd}$

Sønderby, L. C., \& Wagoner, B. (2013). Loneliness: An integrative approach. Journal of Integrated Social Sciences, 3, 1-29.

Sullivan, H. S. (1953). The interpersonal theory of psychiatry. New York, NY: Tavistock Press.

Taylor, R., Conway, L., Calderwood, L., Lessof, C., Cheshire, H., Cox, K., \& Scholes, S. (2007). Health, wealth and lifestyles of the older population in England: The 2002 English Longitudinal Study of Ageing: Technical report. Retrieved from https://www.ifs.org.uk/elsa/report03/ w1_tech.pdf

Valtorta, N., Kanaan, M., Gilbody, S., Ronzi, S., \& Hanratty, B. (2016). Loneliness and social isolation as risk factors for coronary heart disease and stroke: Systematic review and meta-analysis of longitudinal observation studies. Heart, 102, 1009-1016.

Verghese, J., LeValley, A., Derby, C., Kuslansky, G., Katz, M., Hall, C., . . . Lipton, R. B. (2006). Leisure activities and the risk of amnestic mild cognitive impairment in the elderly. Neurology, 66, 821-827. http://dx .doi.org/10.1212/01.wnl.0000202520.68987.48

Weiss, R. (1973). Loneliness: The experience of emotional and social isolation. Cambridge, MA: MIT Press.

Weiss, R. (1974). The provision of social relationships. In Z. Rubin (Ed.), Doing unto others (pp. 17-26). Englewood Cliffs, NJ: Prentice Hall.

Wight, D., Wimbush, E., Jepson, R., \& Doi, L. (2015). Six steps in quality intervention development (6SQuID). Journal of Epidemiology \& Community Health, 70, 520-525. http://dx.doi.org/10.1136/jech-2015205952

Wilson, R. S., Krueger, K. R., Arnold, S. E., Schneider, J. A., Kelly, J. F., Barnes, L. L., . . . Bennett, D. A. (2007). Loneliness and risk of Alzheimer disease. Archives of General Psychiatry, 64, 234-240. http:// dx.doi.org/10.1001/archpsyc.64.2.234

Zunzunegui, M. V., Alvarado, B. E., Del Ser, T., \& Otero, A. (2003). Social networks, social integration, and social engagement determine cognitive decline in community-dwelling Spanish older adults. Journals of Gerontology: Series B, Psychological Sciences and Social Sciences, 58(2), S93-S100. http://dx.doi.org/10.1093/geronb/58.2.S93

Received January 19, 2018

Revision received June 22, 2018 Accepted July 6, 2018 